\title{
Professional flossing as a diagnostic method for gingivitis in the primary dentition
}

\section{Adriela Azevedo Souza Mariath(a) Ana Eliza Lemes Bressani(a) Alex Nogueira Haas ${ }^{(b)}$ Fernando Borba de Araujo(c) Cassiano Kuchenbecker Rösing ${ }^{(\mathrm{d})}$}

(a) MScs, Department of Pediatric Dentistry; (b) MSc, Professor, Department of Periodontology; (c) PhD, Professor, Department of Pediatric Dentistry; (d) PhD, Professor, Department of Periodontology - School of Dentistry, Federal University of Rio Grande do Sul, Porto Alegre, RS, Brazil.

\begin{abstract}
The aim of this study was to evaluate flossing as a diagnostic method for interproximal gingival bleeding in children. For this crossover study, 23 pre-schoolchildren presenting neither restorations nor approximal carious cavities and with at least $15 \%$ of gingival bleeding sites were selected. Examinations were performed at three different moments (3-4 days interval). Examinations comprised repeated measurements of two gingival indices with a 10-minute interval in the following sequences: the Ainamo \& Bay Gingival Bleeding Index (GBI) followed by the Carter \& Barnes flossing index (CBI); CBI followed by GBI; and GBI followed by GBI. Data analysis was performed only for the interproximal sites, considering the GBI as the gold-standard. Agreement between indices, sensitivity (SE), specificity (SP), positive (PPV) and negative predictive values (NPV) were estimated. Percentage agreements in sequences GBI-CBI, CBI-GBI and GBI-GBI were $70.3 \%$, $76.4 \%$ and $84.5 \%$, respectively. Validation of flossing in the first sequence (GBI-CBI) resulted in values of $0.61(95 \% \mathrm{CI} 0.53-0.68), 0.72$ (95\% CI $0.69-0.76), 0.33$ $(95 \% \mathrm{CI} 0.28-0.39)$ and $0.89(95 \% \mathrm{CI} 0.86-0.92)$ respectively for SE, SP, PPV and NPV. It can be concluded that professional flossing is a useful tool in the diagnosis of interproximal gingival inflammatory status in children, especially in conditions of gingival health.
\end{abstract}

Descriptors: Gingivitis; Diagnosis; Dentition, primary; Dental plaque.

\author{
Corresponding author: \\ Adriela Azevedo Souza Mariath \\ Av. Alegrete, 305 - Ap. 401 - Petrópolis \\ Porto Alegre - RS - Brazil \\ CEP: 90460-100 \\ E-mail:adriela.mariath@uol.com.br
}

Received for publication on Oct 23, 2006

Accepted for publication on Aug 03, 2007 


\section{Introduction}

Evaluation of the periodontal status is fundamental during oral health follow-ups of infant patients. The periodontal status is not limited to a periodontal diagnosis, but also provides an assessment of the quality and the routine of the mechanical plaque control performed. Studies have collected information on oral hygiene habits either by means of reported behavior or more directly by using plaque or gingival indices. ${ }^{1}$

It was demonstrated that prevalence, extent and severity of gingivitis increase with age, starting in the primary dentition and reaching their peak during adolescence. ${ }^{2,3}$ Gingivitis in children was also shown to be less severe compared to adults when similar amounts of plaque deposition are found. However, the same gingival indices have been used to diagnose the gingival status in children and adults. ${ }^{4,5}$

The most frequently used clinical indices for evaluation of gingival health have been the Plaque Index (PII $)^{6}$ and the Gingival Index (GI). ${ }^{7}$ The Plaque Index evaluates the presence of plaque attributing four scores according to the plaque volume present on dental surfaces. The Gingival Index associates visual inspection with bleeding of the gingival margin for the diagnosis of the inflammatory condition of the gingiva.

Ainamo, $\mathrm{Bay}^{8}$ (1975) suggested the use of two dichotomous indices. The first, called Visible Plaque Index, aggregates PII scores 0 and 1 as absence and scores 2 and 3 as presence of visible plaque. ${ }^{8}$ Similarly, a dichotomous index to evaluate gingival inflammation was proposed (Gingival Bleeding Index - GBI). The rationale for these dichotomizations is the high subjectivity of quantified classifications of plaque and gingival inflammation. Additionally, it was demonstrated that visual clinical manifestations of gingivitis (edema and gingival redness) is preceded by bleeding of the gingival margin, ${ }^{9}$ consequently the sequence of GI scores would generate some misleading interpretation of the gingival inflammatory condition. Moreover, gingival bleeding was related to histological inflammatory evaluations of the gingival tissues. ${ }^{10}$ Another important consideration related to dichotomous indices of gingivitis is their higher reproducibility. ${ }^{9}, 11$
Specific indices for evaluation of interproximal gingival inflammation have also been suggested. ${ }^{11-14}$ The Carter, Barnes ${ }^{11}$ (1974) gingival bleeding index (CBI) applies dental floss to mechanically stimulate the gingival sulcus aiming at evaluating the absence or presence of bleeding. The authors affirm there is no evidence of traumatic injury of the gingival margin that could lead to a higher occurrence of bleeding. ${ }^{11}$ Loesche $^{13}$ (1979) also introduced a different system, the papillary bleeding score (PBS), in which bleeding is evaluated after the insertion of a Stimudent $^{\circledR}$ interdental cleaner. Tinoco, Gjermo ${ }^{15}$ (1992) observed that flossing was able to detect expected changes in gingival health after plaque control in children 4 to 6 years of age and demonstrated higher sensitivity than GI and GBI to identify reductions in gingival inflammation.

Different indices are available to be applied in the evaluation of gingival inflammation. However, a method that could allow an evaluation of gingival inflammation combining control of dental plaque, oral hygiene instruction and motivation at the same time would be of great value. Such features, associated with easiness of assessment, are desirable characteristics of an index, mainly when applied in infant patients that can present difficult behavior during dental appointments. Thus, the aim of the present study was to evaluate the validity of dental floss as a diagnostic method for gingivitis in the deciduous dentition.

\section{Material and Methods Study design and sample}

A crossover study was conducted with preschool children (3 to 6 years of age) from a Public School from Porto Alegre (Southern Brazil). The inclusion criteria comprised good general health (no pharmacological or medical treatment); absence of proximal caries lesions or restorations detected by visual inspection that could interfere with mechanical plaque control; percentage of gingival bleeding $^{8} \geq 15 \%$; and presence of a complete primary dentition with spaces between all anterior teeth. After screening examinations GBI recording in 49 children, 23 fulfilled the inclusion criteria and were included in the study. 


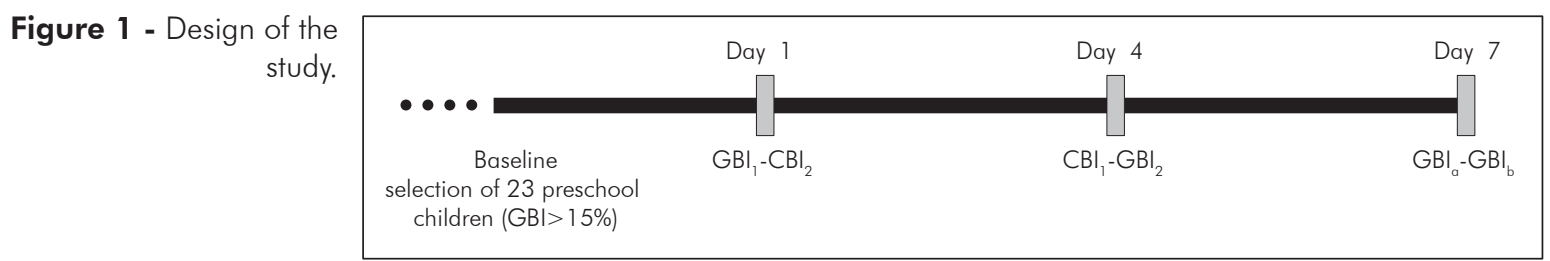

This research is in accordance with the Declaration of Helsinki and was approved by the Ethics Committee, School of Dentistry, Federal University of Rio Grande do Sul, Brazil. Patients were included in this study after parental acceptance and confirmation through a signed Informed Consent.

\section{Procedures}

The study design consisted of three examination sequences performed in all children with an interval period of 3-4 days (Figure 1). These sequences comprised assessment of two gingival indices with a 10minute interval between them, as follows:

- Sequence $1\left(\mathrm{GBI}_{1}-\mathrm{CBI}_{2}\right)$ - Ainamo, Bay ${ }^{8}$ (1975) Gingival Bleeding Index $\left(\mathrm{GBI}_{1}\right)$ recorded in four sites of all teeth present (mesial, buccal, distal, palatal/lingual) followed by Carter, Barnes ${ }^{11}$ (1974) Index $\left(\mathrm{CBI}_{2}\right)$ recorded for all interproximal surfaces;

- Sequence $2\left(\mathrm{CBI}_{1}-\mathrm{GBI}_{2}\right)$ - the same indices were recorded, however in an inverted sequence, CBI followed by GBI;

- Sequence $3\left(\mathrm{GBI}_{\mathrm{a}}-\mathrm{GBI}_{\mathrm{b}}\right)$ - GBI recording was repeated after a 10-minute interval (Figure 1).

\section{Gingival indices}

For both indices, gingival bleeding was recorded as present in a period of 10 seconds after mechanical stimulation of the gingival margin. GBI was recorded using a round sectioned Williams periodontal probe (Neumar, São Paulo, SP, Brazil) and CBI using a dental floss (Sanifill, São Paulo, SP, Brazil).

All examinations were performed by one examiner at school, under natural/artificial light source with the children laying down on class tables.

\section{Examiner reproducibility}

Before starting the study, the reliability for GBI was evaluated with duplicate recordings (3-day in-
Table 1 - Comparison between percentages of bleeding sites in the three examination sequences (mean \pm standard deviation).

\begin{tabular}{|c|c|c|c|}
\hline \multicolumn{2}{|c|}{ Sequences } & $\begin{array}{l}\text { Percentage of } \\
\text { bleeding sites }\end{array}$ & $p$ (Wilcoxon) \\
\hline \multirow{2}{*}{1} & $\mathrm{GBI}_{1}$ & $18.2 \pm 10.7$ & \multirow{2}{*}{0.001} \\
\hline & $\mathrm{CBI}_{2}$ & $33.7 \pm 18.3$ & \\
\hline \multirow{2}{*}{2} & $\mathrm{CBI}_{1}$ & $20.0 \pm 14.6$ & \multirow{2}{*}{0.881} \\
\hline & $\mathrm{GBI}_{2}$ & $19.7 \pm 12.7$ & \\
\hline \multirow{2}{*}{3} & $\mathrm{GBI}_{a}$ & $13.6 \pm 9.8$ & \multirow{2}{*}{0.118} \\
\hline & $\mathrm{GBI}_{\mathrm{b}}$ & $16.3 \pm 11.6$ & \\
\hline
\end{tabular}

terval) of the index in 10 non-participant children. Percentual agreement and kappa coefficient were $85 \%$ and 0.59 , respectively.

\section{Statistical analysis}

Data analysis included only interproximal sites. Validation analysis of CBI was conducted considering GBI as the gold-standard, including a total of 928 interproximal sites. Sensitivity (SE), specificity (SP), positive (PPV) and negative (NPV) predictive values and their respective $95 \%$ confidence intervals $(95 \% \mathrm{CI})$ were calculated. Agreement between the two indices in each of the examination sequences was evaluated calculating total percentage agreement. Wilcoxon signed rank tests were used to compare indices in the same examination sequence. Analysis of variance was conducted to compare the GBI recordings in the three different sequences. The alpha level was set at $5 \%$.

\section{Results}

The mean percentage of interproximal gingival bleeding was $18.2 \% \pm 10.6 \%$ in the first examination sequence (Table 1, GBI $)$. A significant increase in the percentage of bleeding sites was observed when CBI was recorded after GBI (sequence 1, GBI 
Table 2 - Frequency distribution of bleeding sites with GBI followed by CBI.

\begin{tabular}{c|c|c|c}
\hline \multicolumn{2}{c|}{} & \multicolumn{2}{c}{$\mathrm{GBI}_{1}$} \\
\cline { 3 - 4 } \multicolumn{2}{c|}{} & Present & Absent \\
\hline \multirow{2}{*}{$\mathrm{CBI}_{2}$} & Present & 103 & 209 \\
\cline { 2 - 4 } & Absent & 67 & 549 \\
\hline
\end{tabular}

Table 3 - Frequency distribution of bleeding sites with $C B$ followed by GBI.

\begin{tabular}{c|c|c|c}
\hline \multicolumn{2}{c}{} & \multicolumn{2}{c}{$\mathrm{GBI}_{2}$} \\
\cline { 3 - 4 } \multicolumn{2}{c|}{} & Present & Absent \\
\hline \multirow{2}{*}{$\mathrm{CBI}_{1}$} & Present & 73 & 110 \\
\cline { 2 - 4 } & Absent & 109 & 636 \\
\hline
\end{tabular}

Table 4 - Sensitivity, specificity, positive and negative predictive values for $C B I$ recorded after $\left(C B I_{2}\right)$ and before $\left(C B I_{1}\right) G B I$ in sequences 1 and 2 (95\% confidence interval).

\begin{tabular}{c|c|c|c|c}
\hline & Sensitivity & Specificity & Positive predictive value & Negative predictive value \\
\hline $\mathrm{CBI}_{2}$ & $0.61(0.53-0.68)$ & $0.72(0.69-0.76)$ & $0.33(0.28-0.39)$ & $0.89(0.86-0.92)$ \\
\hline $\mathrm{CBI}{ }_{1}$ & $0.40(0.33-0.48)$ & $0.85(0.83-0.88)$ & $0.40(0.33-0.47)$ & $0.85(0.83-0.88)$ \\
\hline
\end{tabular}

Table 5 - Percentual agreement between indices in the three sequences.

\begin{tabular}{c|c}
\hline Sequences & Percentual agreement \\
\hline $1-\mathrm{GBI}_{1} / \mathrm{CBI}_{2}$ & 70.3 \\
\hline $2-\mathrm{CBI}_{1} / \mathrm{GBI}_{2}$ & 76.4 \\
\hline $3-\mathrm{GBI}_{\mathrm{a}} / \mathrm{GBI}_{\mathrm{b}}$ & 84.5 \\
\hline
\end{tabular}

$18.2 \% \pm 10.7 \%$ and $\left.\mathrm{CBI}_{2} 33.7 \% \pm 18.3 \%\right)$. When CBI was recorded before GBI, the percentages of bleeding sites were very similar $(20.0 \% \pm 14.6 \%$ and $19.7 \% \pm 12.7 \%$, respectively) and there was no significant difference between the two indices. In the third sequence, there was a higher percentage of interproximal bleeding sites after the second time GBI was recorded $\left(\mathrm{GBI}_{\mathrm{b}} 16.3 \% \pm 11.6 \%\right)$ than after the first recording (GBI $13.6 \% \pm 9.8 \%$ ), without statistically significant difference between the two examinations. There was no significant difference between the percentages of bleeding sites recorded with GBI in the three examination sequences (ANOVA, $\mathrm{p}=0.163$ ).

Tables 2 and 3 illustrate the frequency distribution of gingival bleeding after GBI and CBI recording in examination sequences 1 and 2, respectively.

Sensitivity, specificity, positive and negative predictive values were calculated and are expressed in Table 4. Specificity of CBI recorded before $\left(\mathrm{CBI}_{1}\right)$ and after $\left(\mathrm{CBI}_{2}\right) \mathrm{GBI}$ was 0.85 and 0.72 , respective- ly. Values of sensitivity for the respective sequences were 0.40 and 0.61 . In relation to predictive values, the chances of a true result with the use of dental floss (CBI) in the absence of disease (absence of gingival inflammation detected by GBI) were $85 \%$ and $89 \%$ (negative predictive values presented in Table 4) when the dental floss was used before and after GBI had been recorded, respectively.

A perfect agreement between CBI and GBI was not observed, regardless of whether dental floss was used before $\left(\mathrm{CBI}_{1}\right)$ or after $\left(\mathrm{CBI}_{2}\right) \mathrm{GBI}$ recording. Percentual agreement of the repeated recordings of GBI was $84.5 \%$ (Table 5 ).

\section{Discussion}

The present study tried to validate the use of dental floss (Carter, Barnes ${ }^{11}$ Index) for the diagnosis of gingivitis in the deciduous dentition. One of the most difficult issues in validation studies is the choice of a gold-standard. There is no consensus in the literature about the gold-standard index for evaluation of gingival inflammation in children. The Gingival Index ${ }^{7}$ and the Gingival Bleeding Index ${ }^{8}$ are the indices mostly used in clinical and epidemiological studies. ${ }^{2,16}$ After considering the indices' peculiarities, the GBI was chosen as the goldstandard in the present study because it is characterized by the diagnosis of gingival inflammation by stimulated bleeding, similarly to the Carter, Barnes $^{11}$ (1974) Index. 
A higher percentage of gingival bleeding sites was observed when CBI was assessed after GBI. However, this finding was not confirmed when CBI was assessed before GBI (Table 1). Two possible reasons for that can be speculated. First, the repetition of an index recording after a certain period of time (10 minutes in the present study) can represent a higher occurrence of bleeding as a result of the trauma caused by the two consecutive mechanical stimulations. ${ }^{15}$ Although there was no difference when GBI was repeated (Table 1 , sequence 3 ), it seems reasonable not to discard the possibility of trauma since bleeding after flossing is a result of a much more close contact with the papilla than that occurred after probing the gingival margin. Further investigations with appropriate experimental designs are needed to elucidate the role of repeated mechanical trauma in gingival bleeding overestimation.

Second, there are differences in the recording procedures for the two indices compared in the present investigation. CBI is assessed with a close contact between dental floss and interdental papilla, reaching the interproximal area in a certain point not necessarily accessed by the probe during GBI recording, allowing the diagnosis of inflammation at initial stages. Thilo et al. ${ }^{17}$ (1986) demonstrated that gingival inflammation in the interproximal area begins in the central area of the papilla. This region may not be accessible to the probe at initial stages of gingival inflammation, leading to false negative results.

There is some discussion in the literature about the viability of reproducing gingival indices. Marks et al. $^{14}$ (1993) have evaluated the degree of reproducibility on the subject level of gingival indices and have observed a high variability across different examiners. Additionally, their results indicated that the Papillary Bleeding Index by Loesche demonstrated higher values of reproducibility compared to other indices. Besides the fact that marginal gingival bleeding upon probing is less subjective than gingival visual inspection, ${ }^{10}$ some variables can also be considered subjective when recording probing indices. Probing force and position, probe design and depth of insertion of the probe may be some examples of variables that are difficult to control during assessment of gingival inflammation. ${ }^{18}$ These are some possible explanations for the complexity involved in reproducing gingival indices, including the repeated recordings of GBI performed in the present study in sequence 3 . Thus, high kappa coefficient values $(>0.61)^{19}$ are not expected for replicate recordings of GBI.

Duplicate measures of gingival inflammation also result in an increase in the percentage of gingival bleeding sites, as could be observed in sequences 1 and 3 (Table 1), similarly to the findings of Tinoco, Gjermo $^{15}$ (1992) who observed an increase of 3.9\% in gingival bleeding three days after the first measurement. Besides, in sequence $2\left(\mathrm{CBI}_{1}-\mathrm{GBI}_{2}\right)$ there was no such increase, a fact that reinforces the idea of dental floss reaching an inaccessible area for the probe. Therefore, a new mechanical stimulus produced by the probe did not result in a significant increase of bleeding sites.

Professional flossing (CBI) was demonstrated to be accurate to evaluate the gingival inflammatory condition in the deciduous dentition. As shown in Table 4, specificity and negative predictive value of CBI were satisfactory when it was recorded before and after GBI, while sensitivity and positive predictive value were lower. Therefore, assessing gingival inflammation with dental floss is more reliable in periodontal health than in disease. These lower values for sensitivity and positive predictive value are the result of a high occurrence of false-positives (209) (Table 2).

Analyzing the present findings, it is possible to make a parallel to the data of Tinoco, Gjermo ${ }^{15}$ (1992). The association between Gingival Index, Gingival Bleeding Index (dichotomized modification) and NBP (percentage of papillary units which did not bleed after stimulation by dental floss) in the primary dentition was assessed, and their ability to detect changes in gingival condition were evaluated. After baseline records, the children received professional plaque control (twice a week) during 3 weeks. The indices were assessed 3 days after the last professional cleaning. The correlation coefficient between GI and percentage of bleeding units was 0.93 at baseline. Only NBP showed statistically significant changes after professional plaque control. The 
authors concluded that this modified version of NBP (using dental floss to stimulate bleeding) seemed to be able to detect the expected change in gingival conditions more effectively than the GI and the dichotomized form. Thus, comparing to GBI and GI, they showed that only flossing was able to detect gingival health condition after some weeks of plaque control. These findings support that flossing is more precise or even more sensitive to detect early signs of gingival inflammation.

In addition to the findings of the present study, another favorable aspect of the professional flossing index is the possibility to combine patient motivation and plaque control instruction with profession-

\section{References}

1. Harris R, Nicoll AD, Adair PM, Pine CM. Risk factors for dental caries in young children: a systematic review of the literature. Community Dent Health. 2004;21(1 Suppl):71-85.

2. Jenkins WM, Papapanou PN. Epidemiology of periodontal disease in children and adolescents. Periodontol 2000. 2001;26(1):16-32.

3. Oh TJ, Eber R, Wang HL. Periodontal diseases in the child and adolescent. J Clin Periodontol. 2002;29(5):400-10.

4. Feldens EG, Kramer PF, Feldens CA, Ferreira SH. Distribution of plaque and gingivitis and associated factors in 3- to 5 -yearold Brazilian children. J Dent Child (Chic). 2006;73(1):410.

5. Matsson L, Goldberg P. Gingival inflammatory reaction in children at different ages. J Clin Periodontol. 1985;12(2):98103.

6. Silness J, Loe H. Periodontal Disease in Pregnancy. II. Correlation between Oral Hygiene and Periodontal Condition. Acta Odontol Scand. 1964;22(2):121-35.

7. Loe H, Silness J. Periodontal Disease in Pregnancy. I. Prevalence and Severity. Acta Odontol Scand. 1963;21(5):533-51.

8. Ainamo J, Bay I. Problems and proposals for recording gingivitis and plaque. Int Dent J. 1975;25(4):229-35.

9. Muhlemann HR, Son S. Gingival sulcus bleeding - a leading symptom in initial gingivitis. Helv Odontol Acta. 1971;15(2):107-13.

10. Greenstein G. The role of bleeding upon probing in the diagnosis of periodontal disease. A literature review. J Periodontol. 1984;55(12):684-8.

11. Carter HG, Barnes GP. The Gingival Bleeding Index. J Periodontol. 1974;45(11):801-5. al plaque removal. Motivational factors are some of the greatest problems involved in preventing and treating behavioral diseases ${ }^{20}$ such as dental caries and periodontal diseases. Thus, the control of periodontal and caries health-disease processes should start as early as possible, so children can learn and construct positive and lifelong habits.

\section{Conclusions}

It can be concluded that professional flossing, by means of assessment of the Carter, Barnes ${ }^{11}$ (1974) Index, is a useful tool for the diagnosis of interproximal gingival inflammatory status in children, mainly as an indicator of gingival health.

12. Abrams K, Caton J, Polson A. Histologic comparisons of interproximal gingival tissues related to the presence or absence of bleeding. J Periodontol. 1984;55(11):629-32.

13. Loesche WJ. Clinical and microbiological aspects of chemotherapeutic agents used according to the specific plaque hypothesis. J Dent Res. 1979;58(12):2404-12.

14. Marks RG, Magnusson I, Taylor M, Clouser B, Maruniak J, Clark WB. Evaluation of reliability and reproducibility of dental indices. J Clin Periodontol. 1993;20(1):54-8.

15. Tinoco NM, Gjermo P. Comparison of the effectiveness of three different methods in detection of changes in gingivitis in the primary dentition. Community Dent Oral Epidemiol. 1992;20(2):84-6.

16. Clerehugh V, Tugnait A. Periodontal diseases in children and adolescents: I. Aetiology and diagnosis. Dent Update. 2001;28(5):222-30, 232.

17. Thilo BE, Caton JG, Polson AM, Espeland MA. Cell populations associated with interdental gingival bleeding. J Clin Periodontol. 1986;13(4):324-9.

18. Barnett ML. Suitability of gingival indices for use in therapeutic trials. Is bleeding a sine qua non? J Clin Periodontol. 1996;23(6):582-6.

19. Landis JR, Koch GG. The measurement of observer agreement for categorical data. Biometrics. 1977;33(1):159-74.

20. Thomson WM, Poulton R, Milne BJ, Caspi A, Broughton $\mathrm{JR}$, Ayers KM. Socioeconomic inequalities in oral health in childhood and adulthood in a birth cohort. Community Dent Oral Epidemiol. 2004;32(5):345-53. 